\section{Structure of the Electrocapillary Double Layer and the Overvoltage of Electrolytic Reactions}

IT is generally accepted ${ }^{1,2}$ that the electrolytio double layer plays an important part in the establishment of potential barriers at electrode surfaces. It is difficult, however, to prove this phenomenon experimentally. It is true that, in order to modify the electrocapillary potential, the composition of the solution has to be changed; but this, in turn, throws doubt upon the interpretation of the results ${ }^{3}$.

It is known that the double layer is composed of a rigid part, bound to the electrode by relatively strong adsorption forces, and of a diffuse part, linked to the first by electrostatic forces. The thickness of this diffuse part is approximately proportional to the inverse square root of the ion concentration ${ }^{4}$.

In extremely dilute solutions it is possible to obtain sufficiently extended diffuse layers, so that a laminar movement may destroy their orderly structure. By reducing the electrostatic compensation, such a movement would provoke an increase of the potential of the adsorbed layer and, consequently, a rise of the overpotential if the adsorbed layer constitutes a potential barrier.

In order to carry out electrolysis in a medium of very low concentration, we used as a reactant oxygen dissolved in water of $10^{-7}$ mhos conductivity. A platinum wire of $0.2 \mathrm{~mm}$. diameter, which did not protrude over the surface of the glass, constituted a rotating electrode ${ }^{5}$. The anode was a cylindrical surface surrounding the rotating electrode; its area was $12 \mathrm{~cm} .^{2}$. We may hence consider the anode as unpolarizable with respect to the cathode. In these circumstances, it is observed that, at a given potential, the reduction current of the oxygen is much decreased when the electrode is moving. This effect is only perceptible at low speeds of rotation. At higher speeds the motion reduces the thickness of the 'diffusion layer', thus again increasing the current. The addition of a small quantity of electrolyte suppresses the phenomenon: at concentrations of potassium chloride greater than $2 \times 10^{-4} M$ the effect ceases to be apparent.

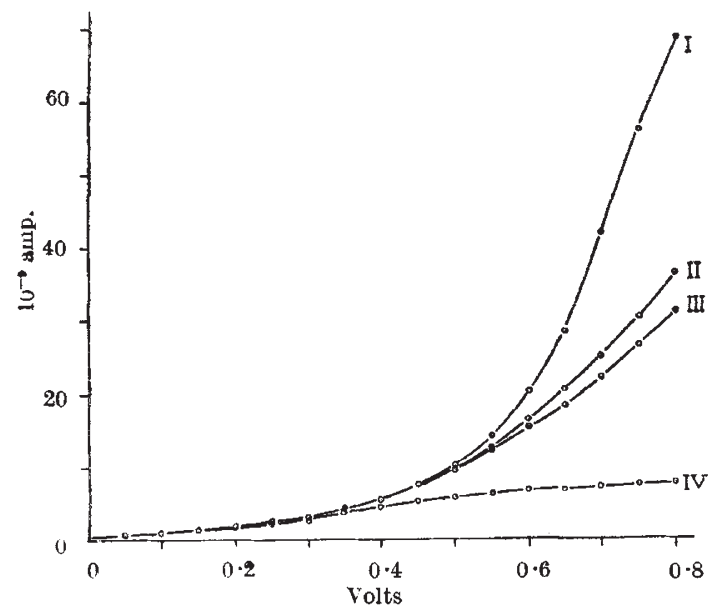

Influence of the electrode movement on the reduction current of oxygen.

Curve I, electrode stationary: curve II, electrode making one revolution in 4 sec.; curve III, electrode making one revolution per sec.; curve IV, residual current in the absence of oxygen;
this current is not influenced by the motion of the electrode
The accompanying graph shows the curves representing the current intensity as a function of the applied potential for different speeds of rotation. The measurements are perfectly reproducible, and we have checked that without polarization the motion of the electrode does not produce any measurable current, even at high speeds of rotation.

\section{J. vaN CAKENBERGHis}

Laboratoire de l'Association pour

les Études Texturales, 4 rue Montoyer,

Brussels.

Nov. 28.

${ }^{1}$ Kimball, G. E., Glasstone, S., and Glassner, A., J. Chem. Phys., 8, 91 (1941).

${ }^{*}$ Frumkin, A., Z. phys. Chem., A, 164, 121 (1933).

se Bethune, A. J., J. Amer. Chem. Soc., 71, 1556 (1949).

- Verwey, E. J. W., and Overbeek, J. Th. G., "Theory of the Stabllity of Lyophobic Colloids", 29 (Amsterdam, 1948).

"Kolthoff, I. M., and Lingane, J. 3., "Polarography", 440 (Now York, 1946).

IN the course of experiments on overpotential at low current densities, during the past four years, we have frequently observed that stirring the solution affects the overpotential. Although our work is far from complete, we now have considerable evidence that, if very great precautions are taken to exclude oxygen and also any products of electrolysis arising from the anode, stirring has little or no effect on the potential of a cathode of a noble metal. For example, some time ago, using cells in which a smooth tungsten or platinum anode was separated from a gold cathode only by a sintered glass plug, stirring caused a considerable lowering of overpotential at the cathode, with polarizing currents less than about ten microamperes per sq. $\mathrm{cm}$. In later work, using very great care over purity of materials and cleanliness of apparatus, and an anode of platinized platinum which could be in the same solution as the cathode, stirring did not affect the potential of cathodes of platinum or gold appreciably. The platinized anode, in the atmosphere of hydrogen, also carefully purified, reduces completely any oxygen produced at the anode, provided that the current is less than about $100 \mu \mathrm{mp} . / \mathrm{cm}^{2}$; this is confirmed by its potential remaining steady at the reversible hydrogen value. With these conditions, stirring had no effect on cathodic potential between $0.05 \mu \mathrm{mp} . / \mathrm{cm} .{ }^{2}$ and $100 \mu \mathrm{amp} . / \mathrm{cm}^{2}{ }^{2}$

The potential of copper cathodes, in $0.1 \mathrm{~N}$ hydrochloric acid, was considerably decreased by stirring, up to $10 \mu \mathrm{mmp} . / \mathrm{cm}^{2}$; at higher currents, stirring had little effect. There was evidence that the copper electrodes had dissolved to a slight extent; we think that stirring assisted diffusion of the previously dissolved copper ions to the cathode, thus increasing the concentration of cations and making it easier for current to pass. At the higher current densities, either the copper ions have been cleared prior to stirring, or the rate of transport of cations under the potential gradient alone is so great that stirring makes little difference.

We think that the changes in the potential for a given current in the presence of oxygen, described by van Cakenberghe, may be due to changes in the concentration of oxygen, or possibly minute traces of surface-active impurities, very near the electrode, where oxygen probably reacts with hydrogen atoms adsorbed on the electrode surface. There seems no 